\title{
In situ and ex situ studies of battery materials with magnetic resonance and diffraction methods
}

\author{
Clare Grey \\ University of Cambridge, Cambridge, United Kingdom; \\ cpg27@cam.ac.uk
}

The development of light, long-lasting rechargeable batteries (and the invention of the lithium-ion battery, now over 25 years ago) has been an integral part of the portable electronics revolution. This revolution has transformed the way in which we communicate and transfer and access data globally. Rechargeable batteries are now playing an increasingly important role in transport and grid applications, but the introduction of these devices comes with different sets of challenges. Importantly, fundamental science is key to producing non-incremental advances and to develop new strategies for energy storage and conversion.

The talk will focus on our work to develop and apply methods that allow devices to be probed while they are operating (i.e., in-situ). This allows the transformations of the various cell components to be followed under realistic conditions without having to disassemble and take apart the cell. To this end, the application of new in and ex-situ Nuclear Magnetic Resonance (NMR), and X-ray diffraction (XRD) approaches to correlate structure and dynamics with function in lithium - and sodium-ion batteries and supercapacitors will be described. To illustrate, we have used NMR, theory and pair distribution function (PDF) analysis methods, to determine the local and longer-range structures of a series of amorphous and disordered $\mathrm{Li}$ and $\mathrm{Na}$ anode structures, including $\mathrm{C}, \mathrm{Sn}, \mathrm{Ge}, \mathrm{Si}$ and $\mathrm{P}$. Both thermodynamic and metastable phases are identified via theoretical (DFT) approaches and compared with NMR, PDF and (in situ) diffraction measurements, the materials often transforming via metastable structures. In the second example, we use in situ X-ray diffraction studies to study the high-rate cycling. Specifically, we are interested in understanding which structural classes of materials can sustain high-rate cycling - and why - and whether the mechanisms for the structural transformations that occur on lithiation/sodiation vary as a function of the rate of battery cycling. Finally, recent work to examine Ni-rich layered cathode materials will be described. These are amongst the most promising candidates for high energy density Li-ion batteries for electric vehicle applications, yet improvements in their capacity retention - particularly under conditions of stress (high/low temperature, fast charging) - are still required for their more widespread use. XRD and NMR spectroscopy are used to understand how Li-ion mobility affects the cycling behaviour of $\mathrm{Li}_{x} \mathrm{Ni}_{0.8} \mathrm{Co}_{0.15} \mathrm{Al}_{0.05} \mathrm{O}_{2}$ and $\mathrm{NMC} 811\left(\mathrm{LiNi}_{0.8} \mathrm{Mn}_{0.1} \mathrm{Co}_{0.1} \mathrm{O}_{2}\right)$. A long duration cell is developed to follow structural changes over multiple battery cycles and over many months.

Keywords: battery materials, nuclear magnetic resonance 